\title{
A deep learning method for delineating early gastric cancer resection margin under chromoendoscopy and white light endoscopy
}

\author{
Ping $\mathrm{An}^{1,2,3} \cdot$ Dongmei Yang ${ }^{1,2,3}$. Jing Wang ${ }^{1,2,3} \cdot$ Lianlian $\mathrm{Wu}^{1,2,3} \cdot$ Jie Zhou ${ }^{1,2,3} \cdot$ Zhi Zeng $^{4} \cdot$ Xu Huang $^{1,2,3}$.

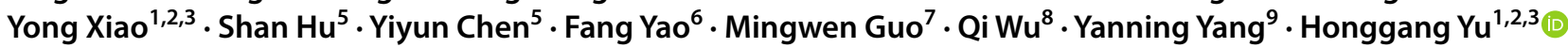

Received: 6 February 2020 / Accepted: 1 April 2020 / Published online: 30 April 2020

(c) The International Gastric Cancer Association and The Japanese Gastric Cancer Association 2020

\begin{abstract}
Background Accurate delineation of cancer margins is critical for endoscopic curative resection. This study aimed to train and validate real-time fully convolutional networks for delineating the resection margin of early gastric cancer (EGC) under indigo carmine chromoendoscopy (CE) or white light endoscopy (WLE), and evaluated its performance and that of magnifying endoscopy with narrow-band imaging (ME-NBI).

Methods We collected CE and WLE images of EGC lesions to train fully convolutional networks ENDOANGEL. ENDOANGEL was tested both on stationary images and endoscopic submucosal dissection (ESD) videos. The accuracy and reliability of ENDOANGEL and NBI-dependent delineation were further evaluated by a novel endoscopy-pathology point-to-point marking.

Results ENDOANGEL had an accuracy of $85.7 \%$ in the CE images and $88.9 \%$ in the WLE images under an overlap ratio threshold of 0.60 in comparison with the manual markers labeled by the experts. In the ESD videos, the resection margins predicted by ENDOANGEL covered all areas of high-grade intraepithelial neoplasia and cancers. The minimum distance between the margins predicted by ENDOANGEL and the histological cancer boundary was $3.44 \pm 1.45 \mathrm{~mm}$ which outperformed the resection margin based on ME-NBI.

Conclusions ENDOANGEL has the potential to assist endoscopists in delineating the resection extent of EGC under CE or WLE during ESD.
\end{abstract}

Keywords Early gastric cancer $\cdot$ Horizontal extent $\cdot$ Fully convolutional networks · Chromoendoscopy $\cdot$ White light endoscopy

\section{Introduction}

Gastric cancer is the third leading cause of cancer death worldwide and is the most common malignancy in east Asian countries [1]. The revolution of the endoscopic

Ping An, Dongmei Yang, Jing Wang contributed equally to this work.

Electronic supplementary material The online version of this article (https://doi.org/10.1007/s10120-020-01071-7) contains supplementary material, which is available to authorized users.

Yanning Yang

ophyyn@163.com

Honggang Yu

yuhonggang@whu.edu.cn

Extended author information available on the last page of the article treatment strategy for early gastric cancer (EGC) using endoscopic submucosal dissection (ESD) dominantly benefits patients with EGC who have the opportunities of en bloc endoscopic resection with shorter hospital stay and a similar 5-year postoperative survival rate compared with the traditional surgery $[2,3]$. This technological advancement has become widespread and is currently recommended as the first-line treatment for EGC without lymph-node metastasis [4].

Accurate delineation of cancer margins is the first critical step in treatment strategy, especially for achieving endoscopic curative resection [5]. Previous studies demonstrated the effectiveness of chromoendoscopy (CE) with an indigo carmine solution after the conventional white-light imaging (WLE) in EGC delineation. Although CE/WLE is widely used in delineating the resection margins of EGC pre-ESD operation $[6,7]$. It still has limitations including 
the inaccuracy for approximately $20 \%$ EGC patients [8]. Recently, magnifying endoscopy with narrow-band imaging (ME-NBI) has been reported as a useful technique in both EGC diagnosis and horizontal delineation of the lesions [9, 10]. However, the conclusions of recent studies comparing ME-NBI and CE in EGC delineation were contradictory [8, 11]. In addition, despite guidelines recommend ME-NBI for delineating the horizontal extent of EGC, the evidence to date still remained insufficient [4].

Artificial intelligence (AI) is one of the fastest growing technologies in recent years. Convolutional neural networks (CNNs) show a remarkable performance in recognizing medical images $[12,13]$. Deep convolutional neural networks (DCNNs) have been successfully used for real-time differentiation of colorectal polyps and classification of skin cancer $[14,15]$. Our previous studies have developed a realtime system for automatically detecting EGC without blind spots during gastroscopy and verified its effect on improving gastroscopy completeness in a randomized-controlled trial $[16,17]$. Till now, the application of CNN in delineating the resection margin of EGC under CE or WLE has rarely been investigated.

In this study, we trained a fully convolutional neural network (FCN) method ENDOANGEL to assist endoscopists in determining the resection extent of EGCs under CE or WLE. Furthermore, we evaluated the performance of ME-NBI and
ENDOANGEL in delineating resection margins using postESD pathology as the gold standard.

\section{Methods}

\section{Image data set and preprocessing}

We collected ESD images obtained from January 1, 2014 and May 1, 2019 in Renmin Hospital of Wuhan University. The data included images of histological confirmed EGC patients with negative lateral margins. Patients who had an undifferentiated EGC, a piecemeal resection, or a positive horizontal margin were excluded. In the study, 1244 images from 536 patients were enrolled, with 889 images from 304 patients for training and the other 355 images from 232 patients for testing. For objectively evaluating the performance of ENDOANGEL, similar images in the test data set were deleted, and only images showing lesions from different perspective were retained. The characteristics of the lesions are shown in Table 1. One expert in EGC, who has performed $>1000 \mathrm{ESD}$, reviewed all the images and delineated the resection margins in the enrolled images without ESD knife markers.

In training data set, $546 \mathrm{CE}$ images from 67 patients were included, and $34 \mathrm{CE}$ images from 14 patients were

Table 1 Characteristics of the lesions

\begin{tabular}{|c|c|c|c|c|c|}
\hline & \multicolumn{2}{|l|}{ Chromoendoscopy } & \multicolumn{2}{|c|}{ White light endoscopy } & \multirow[t]{2}{*}{ Video test set $(n=10)$} \\
\hline & Train set $(n=67)$ & Test set $(n=14)$ & Train set $(n=237)$ & Test set $(n=218)$ & \\
\hline \multicolumn{6}{|c|}{ Tumor location, $n(\%)$} \\
\hline Upper third & $8(11.9)$ & $1(7.1)$ & $23(9.7)$ & $73(33.5)$ & $4(40.0)$ \\
\hline Middle third & $39(58.2)$ & $8(57.2)$ & $126(53.2)$ & $112(51.4)$ & $4(40.0)$ \\
\hline Lower third & $20(29.9)$ & $5(35.7)$ & $88(37.1)$ & $33(15.1)$ & $2(20.0)$ \\
\hline \multicolumn{6}{|c|}{ Macroscopic type, $n(\%)$} \\
\hline Elevated & $31(46.3)$ & $8(57.2)$ & $101(42.6)$ & $46(21.1)$ & $4(60.0)$ \\
\hline Depressed/flat & $36(53.7)$ & $6(42.8)$ & $136(57.4)$ & $172(78.9)$ & $6(60.0)$ \\
\hline \multicolumn{6}{|l|}{ Tumor size, $n(\%)$} \\
\hline$\leq 20 \mathrm{~mm}$ & $47(70.1)$ & $10(71.4)$ & $197(83.1)$ & $188(86.2)$ & $6(60.0)$ \\
\hline$>20 \mathrm{~mm}$ & $20(29.9)$ & $4(28.6)$ & $40(16.9)$ & $30(13.8)$ & $4(40.0)$ \\
\hline \multicolumn{6}{|c|}{ Depth of invasion, $n(\%)$} \\
\hline Mucosa & $36(53.7)$ & $8(57.1)$ & $154(65.0)$ & $170(78.0)$ & $6(60.0)$ \\
\hline Submucosa & $31(46.3)$ & $6(42.9)$ & $83(35.0)$ & $48(22.0)$ & $4(40.0)$ \\
\hline \multicolumn{6}{|c|}{ Histopathologic diagnosis, $n(\%)$} \\
\hline Differentiated & $67(100.0)$ & $14(100.0)$ & 237 (100.0) & $218(100.0)$ & $10(100.0)$ \\
\hline Undifferentiated & $0(0.0)$ & $0(0.0)$ & $0(0.0)$ & $0(0.0)$ & $0(0.0)$ \\
\hline Ulcer findings & $3(4.5)$ & $1(7.1)$ & $11(4.6)$ & $13(6.0)$ & $1(1.0)$ \\
\hline \multicolumn{6}{|l|}{ Facility, $n(\%)$} \\
\hline Olympus & $67(100.0)$ & $14(100.0)$ & $204(86.1)$ & $216(99.1)$ & $10(100.0)$ \\
\hline Fujifilm & $0(0.0)$ & $0(0.0)$ & 33 (13.9) & $2(0.9)$ & $0(0.0)$ \\
\hline
\end{tabular}


included in the test data set. In WLE data set, the train data set consisted of 343 images from 260 patients, and test data set consisted of 321 images from 218 patients. All images were adjusted into $512 \times 512$ pixels. Data were augmented by rotation; scaling; adjusting transparency, and vertical and horizontal directions; and cutting images randomly from 0 to $10 \%$ to improve data set variability and prevent overfitting. The study protocol was approved by the ethics committee of Renmin Hospital of Wuhan University. For retrospective patients whose endoscopic images were stored, informed consent was exempted by the IRB. All prospective patients provided written informed consent before enrollment. All procedures were in accordance with the ethical standards of the responsible committee on human experimentation (institutional and national) and with the Helsinki Declaration of 1964 and later versions.

\section{Training algorithms}

FCNs, which are used for semantic segmentation, were introduced by Jonathan Long and colleagues in 2015. Classification networks are transferred to fully convolutional networks, and their classified information is correspondingly transferred to segmentation. Then, a skip architecture combines semantic information and appearance information to produce segmentation results [18]. UNet++ add redesigned skip pathways and deep supervision to UNet, which meets the need for a more accurate segmentation in medical images [19]. Here, we used UNet++ based on VGG-16 to train the model.

\section{Testing ENDOANGEL in still images}

To investigate the effectiveness of ENDOANGEL in delineating resection margins, we calculated the intersection ratio between the expert and ENDOANGEL delineation regions to the expert delineation region. In the image test data set, manual markers of en bloc resected EGC lesions were histologically demonstrated and, therefore, were chosen as gold standards. The overlap ratio was defined as the ratio of intersection between the expert delineation region and the predicted resection region to the expert delineation region. Threshold was the overlap ratio predefined which ranged from 0.01 to 1.0 with a step size of 0.01 . Exceeding the threshold overlap ratio meant the overlap ratio of a specific case which exceeded the threshold overlap ratio. Correct delineation was defined as the intersection region over the expert delineation region exceeding the threshold overlap ratio. A corrected delineated case was defined as correct delineation of all images of a patient. The accuracy of ENDOANGEL in the test data set was calculated as the number of corrected delineated cases divided by the number of all enrolled cases. To choose an appropriate value to describe the accuracy of ENDOANGEL, two experts were asked to review the results predicted by ENDOANGEL and chose those acceptable results with the overlap value blinded. Through this method, the threshold of 0.6 was chosen to describe the accuracy of ENDOANGEL.

\section{Testing ENDOANGEL in unprocessed ESD videos}

Ten ESD videos with endoscopy-pathology point-to-point markings and post-ESD ME-NBI observations were retrospectively (seven videos) and prospectively (three videos) collected to test the accuracy of ENDOANGEL in real-time delineating the resection margins of EGC. Images were processed and analyzed at 25 frames per second. The blue dotted line indicated the predicting extent of the resection margins (Fig. 2c; Video 1). The video test set was collected retrospectively and the observations were controlled by the endoscopists. Although the application of the ENDOANGEL was presented, a refined analysis was hard to perform because of the quick movements in some endoscopic video clips. We then clipped the video to the sequential images and calculated the accuracy. The gold standard in the video test dataset was the pathological results rebuilt post-ESD. The point-to-point markings by the endoscopists and pathologists were performed as follows:

1. Management of the post-endoscopic resection pathological samples

The specimen was flattened, pinned with a 2-mm pitch on a plastic plate, with appropriate force to flatten the original shape and not to destroy the border of the specimen. After labeling oral or anal border, position, and other information, the plate was immersed in saline followed by ME-NBI as reported previously [20]. The endoscopists examined the integrity of the lesion and marked the suspected region with silk threads. Pins were used to fix the ends of the silk thread. The suspicious cancerous fields were framed by silk threads with color marked by a marker pen (Fig. 1a). Then, the specimen was fixed in $10 \%$ buffered formalin solution (Fig. 1b). The pathologists painted the marked area black with Indian ink (Phygene, China; Fig. 1b). Pathological diagnosis was categorized according to the revised Vienna classification $(\mathrm{C} 1$ : negative for neoplasia; $\mathrm{C} 2$ : indefinite for neoplasia; C3: mucosal low-grade neoplasia [lowgrade adenoma/dysplasia]; $\mathrm{C} 4$ : mucosal high-grade neoplasia [4.1: high-grade adenoma/dysplasia; 4.2: noninvasive carcinoma [carcinoma in situ]; 4.3: intramucosal carcinoma]; and C5: submucosal invasive carcinoma) [21].

2. Rebuilding the pathological results on endoscopic images 


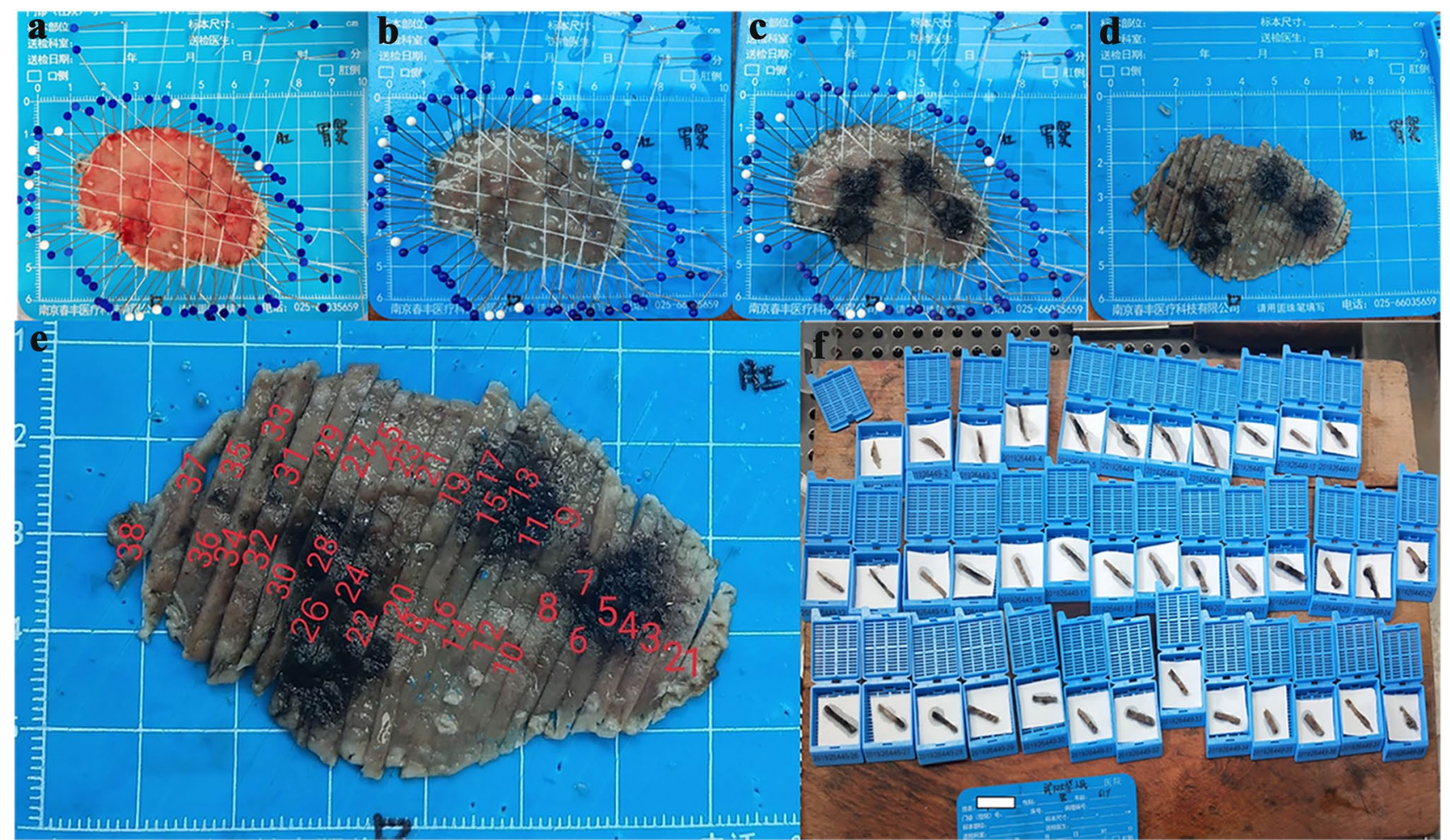

Fig. 1 Marking of the lesion on the specimen. a The resection specimen has undergone post-ESD magnification. A marker pen is used to blacken the silk thread to indicate the suspected lesions. b The fixed specimen. c Application of India ink to suspected lesions. d The specimen is cut into pieces with a $2-\mathrm{mm}$ pitch. e Numbering cut specimen. $\mathbf{f}$ Specimens are placed in the corresponding box
A pathologist rebuilt the pathological results on the formalin-fixed specimen images according to the guidelines and then rebuilt the pathological results on endoscopic images using the ERDAS IMAGINE 9.2 software $[22,23]$. The function was achieved by establishing the equation. The coordinates of six matching points (12 points for two images) were used to establish the equation. Six points were required in one image according to the specification, regardless of the size of the lesion.

(1) Rebuilding the pathological result to the resected specimen: The specimen shrank after fixing in formalin solution. Six matching points were selected from the fixed and resected specimen images. The six points were evenly distributed (Fig. 2a).

(2) Rebuilding the pathological result on endoscopic images: six matching points were selected from resected specimen and endoscopic images. The six points were evenly distributed. Adobe Photoshop (CC 2019) was used to display the lines of the pathological results on endoscopic images (Fig. 2b).

\section{Comparison of the minimum distance between the labeled margins and the pathological cancerous boundary on resection specimen images}

An expert delineated the resection margins according to the post-ESD ME-NBI images, and then, the markers of postESD ME-NBI images were rebuilt to the resected specimen with a measurable scale as above steps. The minimum distance between the cancerous boundary and the marks was determined by calculating the pixel length (Fig. 2d).

\section{Statistical analysis}

Positive predictive value (PPV = true-positive region/[truepositive region + false-positive region]), sensitivity (truepositive region/positive region), and intersection over union (IoU $=$ true-positive/false-positive + true-positive + falsenegative) were calculated to evaluate the performance of ENDOANGEL on the image and video data set [24]. A two-tailed unpaired Student's $t$ test with a significance level of 0.05 was used to compare the minimum distance of the margins and cancerous boundary. A two-tailed unpaired Student's $t$ test with a significance level of 0.05 was used to compare the average area covered by the ENDOANGEL and 

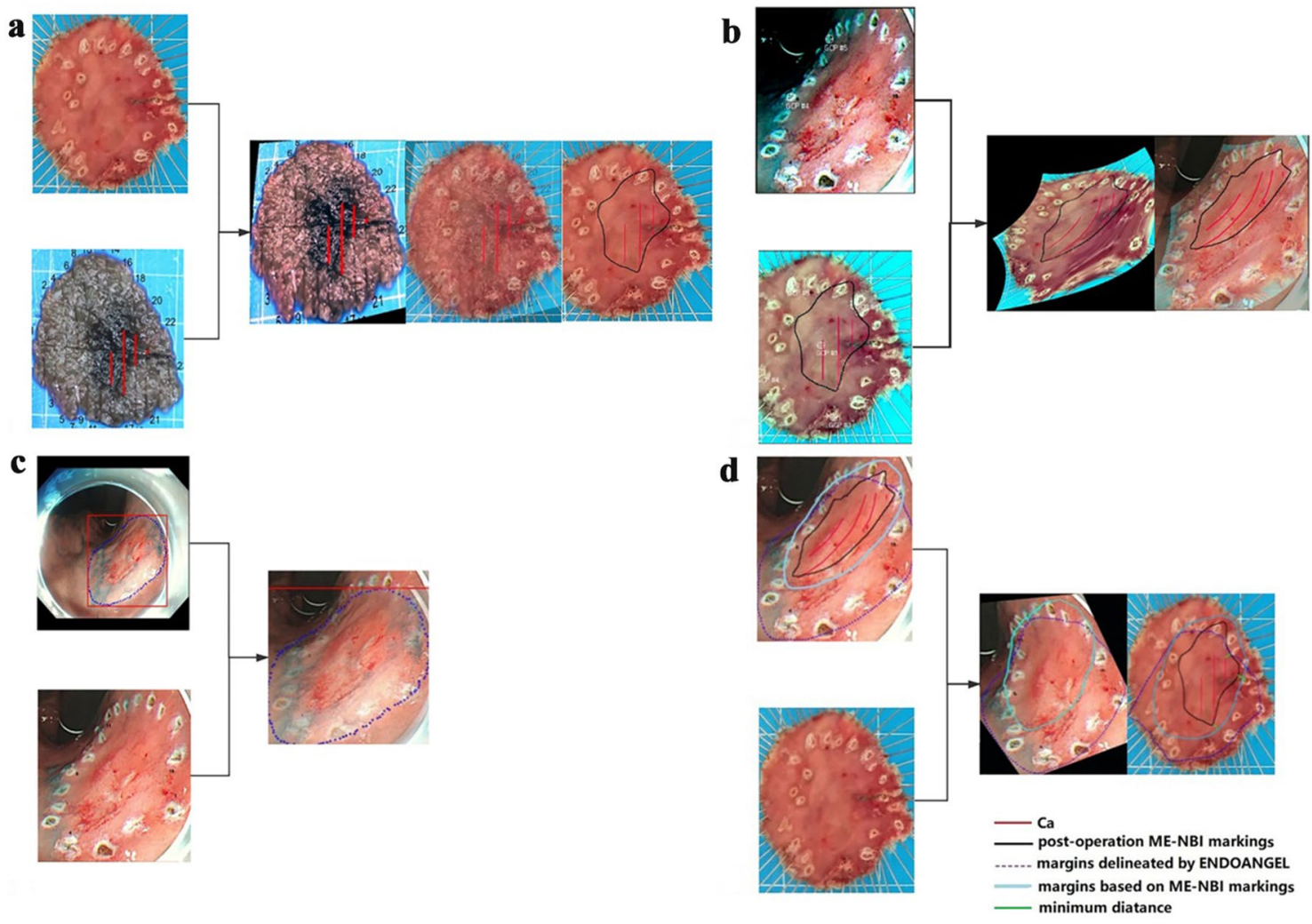

Fig. 2 Process of rebuilding the pathological result on the endoscopic image and rebuilding the endoscopic markings on the resection specimen image. a Step 1: Rebuild the pathological result on the resection specimen image. b Step 2: Rebuild the pathological result on the endoscopic image. The six corresponding points are shown in the images. The change of image's color is caused by the ERDAS IMAG-

ESD knifes. All the calculations were performed with SPSS 20 (IBM, Chicago, IL, USA).

\section{Results}

\section{Endoscopic characteristics of the lesions}

Lesion characteristics are shown in Table 1. All data for a single patient were assigned to exactly one of training or testing splits. The groups were balanced in terms of tumor size, location, and macroscopic type.

\section{Performance of ENDOANGEL in delineating resection margins in stationary images}

In Fig. 3, the accuracy of ENDOANGEL was shown under a series of overlap ratio ranging from 0.01 to 1.0 with a step size of 0.01 . Compared with the manual markings by the expert, ENDOANGEL had an accuracy of $85.7 \%$ in the CE images and $88.9 \%$ in the WLE images under an overlap ratio
INE 9.2 software, which does not affect the rebuild process. c Step 3: Present the extent delineated by ENDOANGEL on the endoscopic image and present different marks on the endoscopic image. d Step 4: Present the marks on the resection specimen image. Rebuild margins based on the postoperative ME-NBI markings on the resection specimen image, and then mark the minimum distance

threshold of 0.60 . The average IoU is 0.688 of CE image test data set and 0.668 of WLE image test data set. Representative images of ENDOANGEL delineating the resection extent are shown in Fig. 4.

\section{Performance of ENDOANGEL in unprocessed ESD videos}

Compared with ESD knife markings, ENDOANGEL reached an accuracy of $100 \%$ under an overlap ratio threshold of 0.60 (Table 2). Compared with pathological rebuilt results, ENDOANGEL and ESD knife marked regions both covered all areas of high-grade intraepithelial neoplasia and cancers. The average area covered by ENDOANGEL and ESD Knife had no difference $(p=0.33)$. The minimum distance between the labeled margins and pathological cancerous boundary was $3.40 \pm 1.49 \mathrm{~mm}$ for ENDOANGEL and $3.32 \pm 2.32 \mathrm{~mm}$ for the ESD knife marking by the experts, with no significant difference between them $(p=0.32)$. A representative video of ENDOANGEL delineating the resection extent in real time was presented in Video 1. 
Fig. 3 Performance of ENDOANGEL in delineating the resection margin in the test dataset. a Chromoendoscopy image data set. b White-light endoscopy image data set
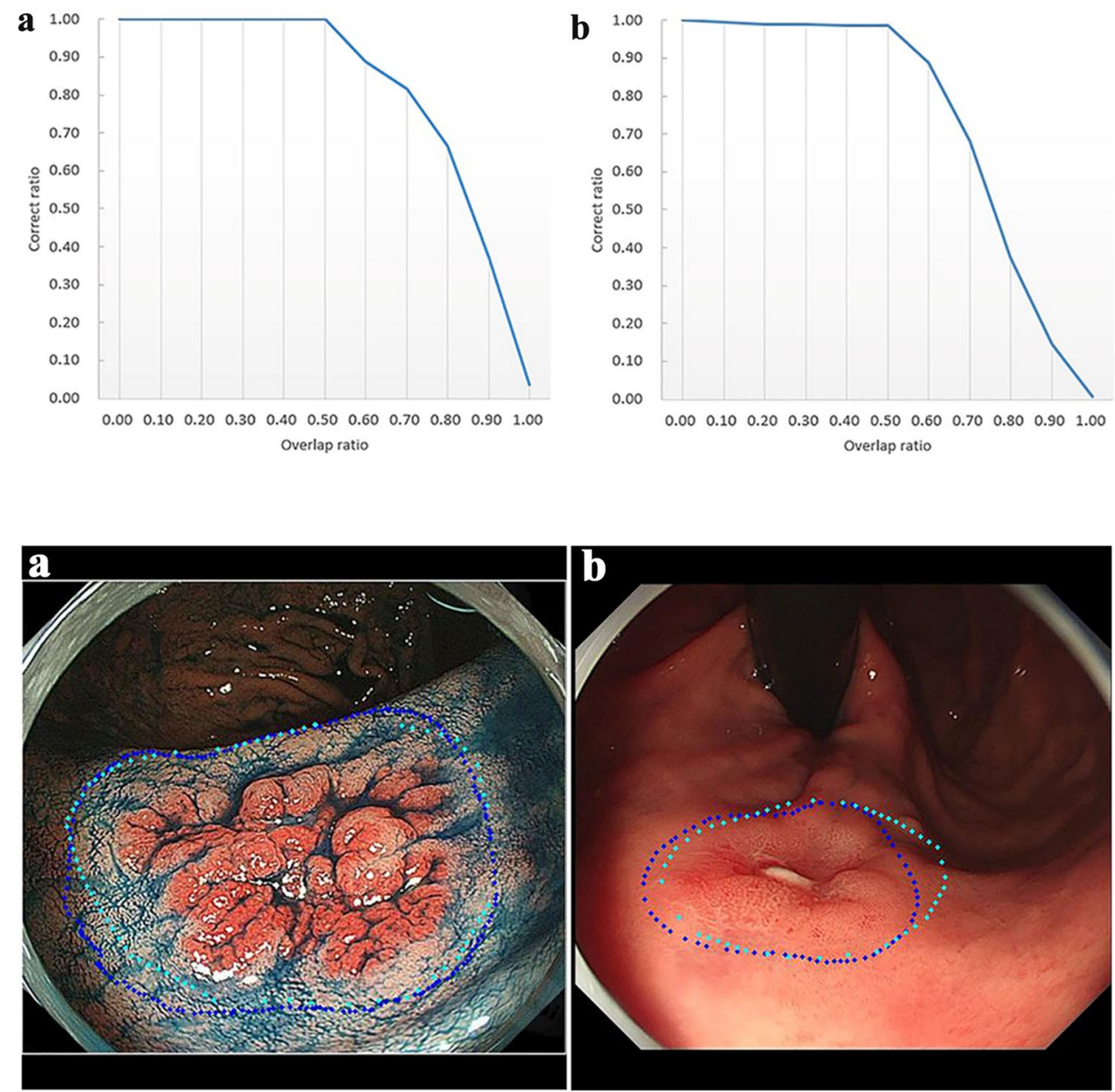

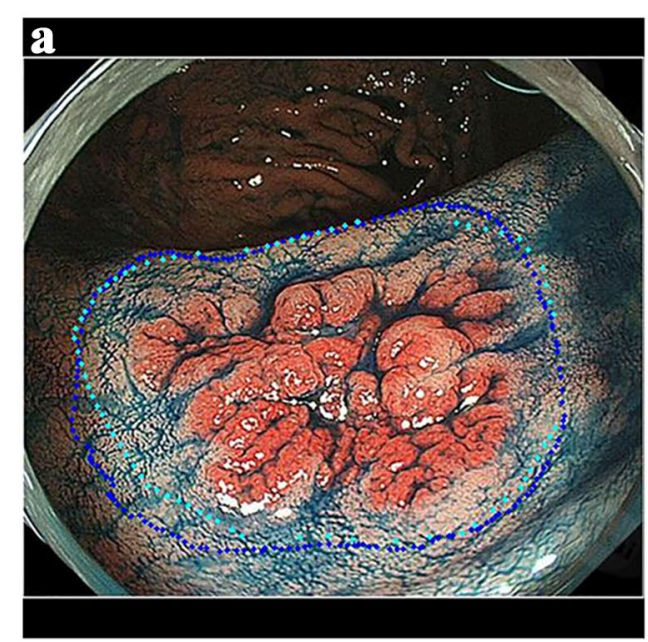

Fig. 4 Representative images of ENDOANGEL delineating the resection extent. The dark dotted line is the resection margin predicted by ENDOANGEL. The light dotted line is the resection margin delineated by the expert. a ENDOANGEL delineates the resection extent in the CE images. The overlap ratio is $98.79 \%$. b ENDOANGEL delineates the resection extent in the WLE images. The overlap ratio is $89.49 \%$
Table 2 Performance of ENDOANGEL in image data set and video data set in comparison with that of manual markings by experts

\begin{tabular}{llll}
\hline & IoU & Sensitivity & PPV \\
\hline Image data set & $67.6(7.5)$ & $81.7(8.5)$ & $83.8(7.4)$ \\
Video data set & $70.4(6.9)$ & $89.5(5.5)$ & $76.6(5.2)$ \\
\hline
\end{tabular}

All results are given as percentage (standard deviation)

$I o U$ intersection over union, $P P V$ positive predictive value

\section{Evaluation of ME-NBI and ENDOANGEL in delineating resection margins by post-ESD pathology}

In ten cases with endoscopy-pathology point-to-point markings, the resection margins predicted by ENDOANGEL covered all areas of high-grade intraepithelial neoplasia and cancers, while the resection margins based on ME-NBI only covered all cancerous regions in $80.00 \%(8 / 10)$ of the patients. Furthermore, for eight cases with negative lateral margins determined by ME-NBI, the minimum distance between the predicted margins and pathological cancerous boundary was $3.44 \pm 1.45 \mathrm{~mm}$ for ENDOANGEL and slightly shorter for ME-NBI $(3.21 \pm 1.72 \mathrm{~mm}, p=0.80)$. Representative images of the performance of ME-NBI and ENAOANGEL in delineating resection margins are shown in Fig. 2c.

\section{Discussion}

Precise evaluation of the lateral margins of EGCs is vital for curable resection and reducing complications. WLE combined with $\mathrm{CE}$, which enhances the characteristics of lesions, has been widely used for delineating the horizontal margins of EGCs [25, 26]. Recently, ME-NBI, which determines the demarcation line by observing the microvasculature and microsurface, has also been recommended $[9,10]$. Several studies have investigated and compared the effectiveness of ME-NBI and CE in delineating EGC. However, their conclusions were contradictory $[8,11]$. Here, we developed a novel method to objectively evaluate the performance of post-ESD ME-NBI in delineating EGC margins and built a deep learning-based method to identify the resection extent 
of EGC under CE or WLE both in images and sequential images clipped from real-time EGC videos.

Although pathological analysis was routinely performed after ESD, direct comparison between the pathological characteristics and endoscopic lesion appearances is still challenging for both pathologists and endoscopists. Researchers matched pathological results and lesions under endoscopy mainly according to the typical changes such as noticeable protuberance and indentation [27]. Nagahama et al. also studied EGC resection margins. They took two biopsies $5 \mathrm{~mm}$ outside and inside the oral-side margins to compare the capacity of ME-NBI and CE in delineating the extent of EGC [8]. The main limitations included that the small biopsy specimen could not represent the extent of the whole resected specimen, and sampling errors and subjective deviations increased the possible discrepancies [8]. Here, we first developed a novel method to recover post-ESD from endoscopic views which achieved point-to-point matching between endoscopy and pathology, and provided an objective and visualized method to evaluate the performance in different endoscopy modalities. More importantly, through this method, we revealed that post-ESD ME-NBI only covered whole cancerous regions in $80.00 \%$ (8/10) of the patients, and the distance of the cutting edge was slightly short even in negative-margin cases. These findings were finally reconfirmed by pathological rebuilding. In another two cases, post-ESD ME-NBI predicting EGC margins were assessed as positive when compared with rebuilt pathological images. The previous studies in undifferentiated cancers revealed that in-depth infiltration of cancer cells and with non-neoplastic epithelium covering might cause ME-NBI misdiagnosis [28]. However, in the current studies, pathological results of these two cases with positive margins predicted by post-ESD ME-NBI excluded this possibility, because they were both surficial, differentiated EGCs and without any in-depth invasion. It was not clear whether the ME-NBI which was made on the resected specimens might account for the difference. Further studies need to investigate the ability of ME-NBI in determining EGC margins objectively and provide more practical evidence for clinical treatments.

A previous study tried to use a computer-aided system to diagnose and delineate EGCs in retrospective ME-NBI images [29]. However, little studies focused on delineating EGC margins under CE or WLE. Based on our previous work on $\mathrm{AI}$ in EGC, we developed a deep learning method ENDOANGEL to identify the resection extent of EGC under CE or WLE $[16,17]$. Its performance was comparable with that of the expert both in stationary images and real-time videos. In the pathologically rebuilt endoscopic cases, ENDOANGEL completely encircled the cancerous areas with suitable ranges of cutting margin. Furthermore,
ENDOANGEL achieved real-time delineation by analyzing video data at $80 \mathrm{~ms}$ per frame. In contrast, delineating EGC margins by ME-NBI is absolutely time-consuming (a mean operating time of around 45 min per lesion) [30]. The much shorter prediction time and the absence of fatigue with the CNN, ENDOANGEL will not only assist endoscopists in delineating resection margins, but also save a large part of time and energies. The outstanding real-time delineation by ENDOANGEL will also help endoscopists marking EGC lesions in ESD operations.

It is also worth noting that our pathological rebuilt method was greatly facilitated the accurate evaluation of EGC margins. The novel way of rebuilding pathological results on endoscopic images could greatly promote the relocations of endoscopic lesions in pathological cancers, directly match pathological findings with endoscopic characteristics in a point-to-point way, and greatly improve the endoscopists understanding of EGC. These first direct and rational findings will potentially benefit and be applied in future clinical diagnosis, EGC training, and endoscopic cancer studies. In noncurative resection cases, ENDOANGEL is also expected to provide useful information for further endoscopic retreatment.

This study has several limitations. First, we selected patients who underwent en bloc resection to develop ENDOANGEL, which might cause bias. Lesions that were easier to resect were more likely to be enrolled. However, the bias can be reduced by increasing the number of cases. Second, the number of cases with pathological rebuild results in video test set was limited. A large-scale, prospective study needs to further verify the robustness of ENDOANGEL. We are carrying out further prospective and multicenter studies and more patients would be enrolled to verify the model in the future. A system based on ENDOANGEL will also be established, which contains input module, analyzed module, and output module to connect with clinical endoscopic video imaging system. Third, the real-time prediction of ENDOANGEL could be affected by man-made disturbances such as cautery marks. The performance of ENDOANGEL should be further refined with more images to limit the impact.

We trained an AI method ENDOANGEL to delineate resection margins of EGC under CE or WLE and developed an endoscopy-pathology by point-to-point matching for objectively evaluating its performance. ENDOANGEL presented a potential in assisting endoscopists in the delineation of the resection margins for EGC patients.

Funding This study was funded by Project of Hubei Provincial Clinical Research Center for Digestive Disease Minimally Invasive Incision (Grant Number 2018BCC337); Hubei Province Major Science and Technology Innovation Project (Grant Number 2018-916-000-008); the National Natural Science Foundation of China [Grant Number 
81672387 (to Yu Honggang)]; the National Natural Science Foundation of China [Grant Number 81302131(to Ping An)]; the National Natural Science Foundation of China [Grant Number 81770899 (to Yanning Yang)].

\section{Compliance with ethical standards}

Conflict of interest The authors declare that they have no conflict of interest.

\section{References}

1. Bray F, Ferlay J, Soerjomataram I, Siegel RL, Torre LA, Jemal A. Global cancer statistics 2018: GLOBOCAN estimates of incidence and mortality worldwide for 36 cancers in 185 countries. CA Cancer J Clin. 2018;68:394-424.

2. Isomoto H, Shikuwa S, Yamaguchi N, Fukuda E, Ikeda K, Nishiyama $\mathrm{H}$, et al. Endoscopic submucosal dissection for early gastric cancer: a large-scale feasibility study. Gut. 2009;58:331-6.

3. Lee S, Choi KD, Han M, Na HK, Ahn JY, Jung KW, et al. Longterm outcomes of endoscopic submucosal dissection versus surgery in early gastric cancer meeting expanded indication including undifferentiated-type tumors: a criteria-based analysis. Gastric Cancer. 2018;21:490-9.

4. Pimentel-Nunes P, Dinis-Ribeiro M, Ponchon T, Repici A, Vieth M, De CA, et al. Endoscopic submucosal dissection: European Society of Gastrointestinal Endoscopy (ESGE) guideline. Endoscopy. 2015;47:829-54.

5. Bourke MJ, Neuhaus H, Bergman JJ. Endoscopic submucosal dissection: indications and application in western endoscopy practice. Gastroenterology. 2018;154:1887-900.

6. Zhao Z, Yin Z, Wang S, Wang J, Bai B, Qiu Z, et al. Meta-analysis: the diagnostic efficacy of chromoendoscopy for early gastric cancer and premalignant gastric lesions. J Gastroenterol Hepatol. 2016;31:1539-45.

7. Yamashita H, Kitayama J, Ishigami H, Yamada J, Miyato H, Kaisaki $\mathrm{S}$, et al. Endoscopic instillation of indigo carmine dye with acetic acid enables the visualization of distinct margin of superficial gastric lesion; usefulness in endoscopic treatment and diagnosis of gastric cancer. Dig Liver Dis. 2007;39:389-91.

8. Nagahama T, Yao K, Uedo N, Doyama H, Ueo T, Uchita K, et al. Delineation of the extent of early gastric cancer by magnifying narrow-band imaging and chromoendoscopy: a multicenter randomized controlled trial. Endoscopy. 2018;50:566-76.

9. Nagahama T, Yao K, Maki S, Yasaka M, Takaki Y, Matsui T, et al. Usefulness of magnifying endoscopy with narrow-band imaging for determining the horizontal extent of early gastric cancer when there is an unclear margin by chromoendoscopy (with video). Gastrointest Endosc. 2011;74:1259-67.

10. Nonaka K, Namoto M, Kitada H, Shimizu M, Ochiai Y, Togawa $\mathrm{O}$, et al. Usefulness of the DL in ME with NBI for determining the expanded area of early-stage differentiated gastric carcinoma. World J Gastrointest Endosc. 2012;4:362-7.

11. Asada-Hirayama I, Kodashima S, Sakaguchi Y, Ono S, Niimi $\mathrm{K}$, Mochizuki S, et al. Magnifying endoscopy with narrow-band imaging is more accurate for determination of horizontal extent of early gastric cancers than chromoendoscopy. Endosc Int Open. 2016;4:E690-E698698.

12. Simonyan K, Zisserman A. Very deep convolutional networks for large-scale image recognition. arXiv preprint arXiv. 2014. pp. 1409-556.

13. Krizhevsky A, Sutskever I, Hinton G. Imagenet classification with deep convolutional neural networks. Adv Neural Inf Process Syst 2012. pp. 1097-105.
14. Byrne MF, Chapados N, Soudan F, Oertel C, Linares MP, et al. Real-time differentiation of adenomatous and hyperplastic diminutive colorectal polyps during analysis of unaltered videos of standard colonoscopy using a deep learning model. Gut. 2019;68:94-100.

15. Esteva A, Kuprel B, Novoa RA, Ko J, Swetter SM, Blau HM, et al. Dermatologist-level classification of skin cancer with deep neural networks. Nature. 2017;542:115.

16. Wu LL, Zhou W, Wan XY, Zhang J, Shen L, Hu S, et al. A deep neural network improves endoscopic detection of early gastric cancer without blind spots. Endoscopy. 2019;51:522-31.

17. Wu LL, Zhang J, Zhou W, An P, Shen L, Liu J, et al. Randomised controlled trial of WISENSE, a real-time quality improving system for monitoring blind spots during esophagogastroduodenoscopy. Gut. 2019. https://doi.org/10.1136/gutjnl-2018-317366.

18. Long J, Shelhamer E, Darrell T. Fully convolutional networks for semantic segmentation. IEEE Trans Pattern Anal Mach Intell. 2015;2015:645-51.

19. Zhou Z, Siddiquee MMR, Tajbakhsh N, Liang J. UNet++: A Nested U-Net Architecture for Medical Image Segmentation. In: Stoyanov D. et al. (eds.) Deep learning in medical image analysis and multimodal learning for clinical decision support. DLMIA 2018, MLCDS 2018. Lecture notes in computer science, vol 11045. Cham: Springer.

20. Kikuchi D, Iizuka T, Hoteya S, Nomura K, Kuribayashi Y, Toba T, et al. Vascular density of superficial esophageal squamous cell carcinoma determined by direct observation of resected specimen using narrow band imaging with magnifying endoscopy. Dis Esophagus. 2017;30:1-5.

21. Schlemper RJ, Riddell RH, Kato Y, Borchard F, Cooper HS, Dawsey SM, et al. The Vienna classification of gastrointestinal epithelial neoplasia. Gut. 2000;47:251-5.

22. ERDAS. ERDAS field guide: Erdas, 1997.

23. Geosystems L. ERDAS imagine. Atlanta, 2004.

24. Huaizu J, Erik LM. Face Detection with the faster R-CNN. In: 2017 12th IEEE International Conference on Automatic Face and Gesture Recognition (FG 2017); 2017. pp. 650-657.

25. Sakai Y, Eto R, Kasanuki J, Kondo F, Kato K, Arai M, et al. Chromoendoscopy with indigo carmine dye added to acetic acid in the diagnosis of gastric neoplasia: a prospective comparative study. Gastrointest Endosc. 2008;68:635-41.

26. Numata N, Oka S, Tanaka S, Yoshifuku Y, Miwat T, Sanomura Y, et al. Useful condition of chromoendoscopy with indigo carmine and acetic acid for identifying a demarcation line prior to endoscopic submucosal dissection for early gastric cancer. BMC Gastroenterol. 2016;16:72.

27. Makazu M, Hirasawa K, Sato C, Ikeda R, Fukuchi T, Ishii Y, et al. Histological verification of the usefulness of magnifying endoscopy with narrow-band imaging for horizontal margin diagnosis of differentiated-type early gastric cancers. Gastric Cancer. 2018;21:258-66.

28. Yao K, Anagnostopoulos G, Ragunath K. Magnifying endoscopy for diagnosing and delineating early gastric cancer. Endoscopy. 2009;41:462-7.

29. Kanesaka T, Lee TC, Uedo N, Lin KP, Chen HZ, Lee JY, et al. Computer-aided diagnosis for identifying and delineating early gastric cancers in magnifying narrow-band imaging. Gastrointest Endosc. 2018;87:1339-444.

30. Sumiyama K. Past and current trends in endoscopic diagnosis for early stage gastric cancer in Japan. Gastric Cancer. 2017;20:20-7.

Publisher's Note Springer Nature remains neutral with regard to jurisdictional claims in published maps and institutional affiliations. 


\section{Affiliations}

\section{Ping $A n^{1,2,3}$. Dongmei Yang ${ }^{1,2,3}$. Jing Wang ${ }^{1,2,3} \cdot$ Lianlian $\mathrm{Wu}^{1,2,3} \cdot$ Jie Zhou ${ }^{1,2,3} \cdot$ Zhi Zeng $^{4} \cdot \mathrm{Xu} \mathrm{Huang}^{1,2,3}$.

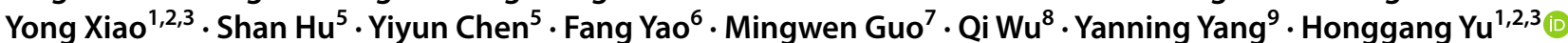

Ping An

anping_05@163.com

Dongmei Yang

1668295897@qq.com

Jing Wang

wjlnsm@gmail.com

Lianlian $\mathrm{Wu}$

wu_leanne@163.com

Jie Zhou

zhoujie0116@163.com

Zhi Zeng

zhizeng@whu.edu.cn

Xu Huang

ahhx1987@qq.com

Yong Xiao

35789825@qq.com

Shan $\mathrm{Hu}$

shan.hu@ai-endoangel.com

Yiyun Chen

chenyy@whu.edu.cn

Fang Yao

yaofang99@sina.com

Mingwen Guo

32583669@qq.com

Qi Wu

wuqi1973@163.com
1 Department of Gastroenterology, Renmin Hospital of Wuhan University, 99 Zhangzhidong Road, Wuhan 430060, Hubei Province, China

2 Key Laboratory of Hubei Province for Digestive System Disease, Renmin Hospital of Wuhan University, Wuhan, China

3 Hubei Provincial Clinical Research Center for Digestive Disease Minimally Invasive Incision, Renmin Hospital of Wuhan University, Wuhan, China

4 Department of Pathology, Renmin Hospital of Wuhan University, Wuhan, China

5 School of Resources and Environmental Sciences of Wuhan University, Wuhan, China

6 Department of Gastroenterology, Peking Union Medical College Hospital, Chinese Academy of Medical Sciences \& Peking Union Medical College, Beijing, China

7 Department of Gastroenterology, The first hospital of Yichang, Yichang, China

8 Department of Endoscopy, Key Laboratory of Carcinogenesis and Translational Research (Ministry of Education), Peking University Cancer Hospital \& Institute, Beijing, China

9 Department of Ophthalmology, Renmin Hospital of Wuhan University, 99 Zhangzhidong Road, Wuhan 430060, Hubei Province, China 